\title{
Les effets des rayonnements ionisants sur le système cardiovasculaire
}

\author{
F. MILLIAT ${ }^{1}$, M. BENDERITTER $^{1}$, M.-H. GAUGLER ${ }^{1}$
}

(Manuscrit reçu le 25 avril 2011, accepté le 19 juillet 2011)

RÉSUMÉ Le traitement par radiothérapie des cancers des sphères thoracique, médiastinale et ORL est associé à un risque de complications cardiovasculaires. Les patients traités et guéris de leur cancer sont de plus en plus nombreux, vivent de plus en plus longtemps, et leur devenir à long terme doit être pris en considération. Les complications cardiovasculaires associées principalement au traitement des cancers du sein, du lymphome de Hodgkin et des tumeurs de la tête et du cou se manifestent de manière insidieuse et chronique. Leur survenue est liée à de nombreux facteurs comme l'âge du patient au moment du traitement, le nombre d'années suivant la radiothérapie, la dose et le volume au cœur et aux gros vaisseaux (artères coronaires et carotides) ou encore l'association avec les autres facteurs de risque cardiovasculaire traditionnels. Les mécanismes physiopathologiques sont mal connus. Même si des similitudes avec l'athérosclérose liée à l'âge sont établies, les spécificités et les particularités de l'athérosclérose radio-induite pour des fortes doses d'irradiation restent à établir. Pour des faibles niveaux d'exposition aux rayonnements ionisants, des études épidémiologiques récentes suggèrent aussi un risque accru de développer des pathologies cardiovasculaires. L'amélioration des connaissances sur les mécanismes lésionnels à l'origine des pathologies cardiovasculaires radio-induites et l'identification plus précise des populations à risque devraient permettre dans le futur une prise en charge plus efficace de ces patients à risque vasculaire.

ABSTRACT Effect of ionizing radiation on cardiovascular system

Radiotherapy treatment for cancer of the chest, mediastinal area or the neck area is associated with increased risk of cardiovascular disease. With the increasing number of cancer patients and the increased treatment efficiency, the number of cancer survivors is increasing exponentially. The cancer survivors live longer and their long-term follow-up must be considered. The cardiovascular toxicity is mainly associated with the treatment of breast cancer, Hodgkin's lymphoma and head and neck cancer. Radiation-induced cardiovascular effects are insidious and chronic. Their occurrence is linked to numerous factors including the age of the patient at the beginning of the radiotherapy schedule, the number of years following radiotherapy, the doses (and volume) to the heart and the large vessels (coronary and carotid arteries), and the association with the traditional cardiovascular risk factors. Pathophysiological mechanisms remain unclear and, even if similarities with agerelated atherosclerosis were established, the specificities of the radiation-induced atherosclerosis for high doses remain to be discovered. For low/moderate doses of ionising radiation, recent epidemiological studies provide evidence of increased risk of cardiovascular pathologies. A better knowledge of the mechanisms associated

\footnotetext{
1 Institut de radioprotection et de sûreté nucléaire, DRPH, SRBE, Laboratoire de radiopathologie et de thérapies expérimentales (LRTE), Fontenay-aux-Roses, France.
} 
with the radiation-induced cardiovascular pathologies and the more precise identification of the populations at risk in the future should allow a more effective care of these patients with cardiovascular risk.

Keywords: Ionising radiation / radiotherapy / cardiovascular risk

\section{Introduction}

Les maladies cardiovasculaires représentent la première cause de mortalité dans le monde (Ross, 1999). Au sens large, ces pathologies sont associées à des dysfonctionnements du système circulatoire et affectent la fonction et/ou l'intégrité de différents organes comme le cœur, le cerveau, les reins ou encore les membres inférieurs. Des facteurs de risque tels que l'hypercholestérolémie, l'hypertension, le diabète, et le tabagisme sont associés à ces pathologies. L'utilisation des rayonnements ionisants en thérapeutique et en diagnostic exposent aussi les patients à un risque de complications cardiovasculaires. Des études épidémiologiques concernant des patients traités par radiothérapie pour un lymphome de Hodgkin, (Swerdlow et al., 2007) pour un cancer du sein (Paszat et al., 1998) ou de la tête et du cou (Dorresteijn et al., 2002) montrent un lien entre l'exposition aux rayonnements ionisants et le risque de développer à plus ou moins long terme des pathologies cardiovasculaires. Pour les fortes doses d'irradiation, des études expérimentales viennent étayer les observations cliniques et apportent des éléments quant aux mécanismes biologiques sous-jacents. Pour des niveaux d'exposition plus faibles, les résultats sont plus contradictoires et sont obtenus sur la base de quelques études épidémiologiques (McGale and Darby, 2005 ; Little et al., 2008 ; Metz-Flamant et al., 2009 ; Little et al., 2010).

Les pathologies cardiovasculaires radio-induites résultent d'atteintes du cœur et des gros vaisseaux (Fig. 1). Les pathologies cardiaques incluent des anomalies cardiaques pouvant atteindre les différentes tuniques du cœur comme le péricarde, le myocarde et l'endocarde et à l'origine de coronaropathies, de valvulopathies symptomatiques ou asymptomatiques. Ces pathologies peuvent aboutir à des insuffisances cardiaques modérées à sévères. Les cardiopathies ischémiques incluent l'infarctus du myocarde et l'angine de poitrine. Au niveau du cœur, il convient de dissocier les complications cardiaques résultant de l'atteinte des microvaisseaux (péricardite, myocardite et endocardite) lors d'irradiation de la sphère thoracique (Heidenreich and Kapoor, 2009), dont le stade ultime est la fibrose cardiaque radique, des complications cardiaques résultant de l'atteinte des artères coronaires (pouvant être à l'origine de cardiopathie ischémiques et d'infarctus du myocarde) lors d'irradiations thérapeutiques pour traiter un lymphome de Hodgkin ou un cancer du sein. Les complications cérébrovasculaires sont, elles, liées à l'atteinte des artères carotides lors de radiothérapie pour des 


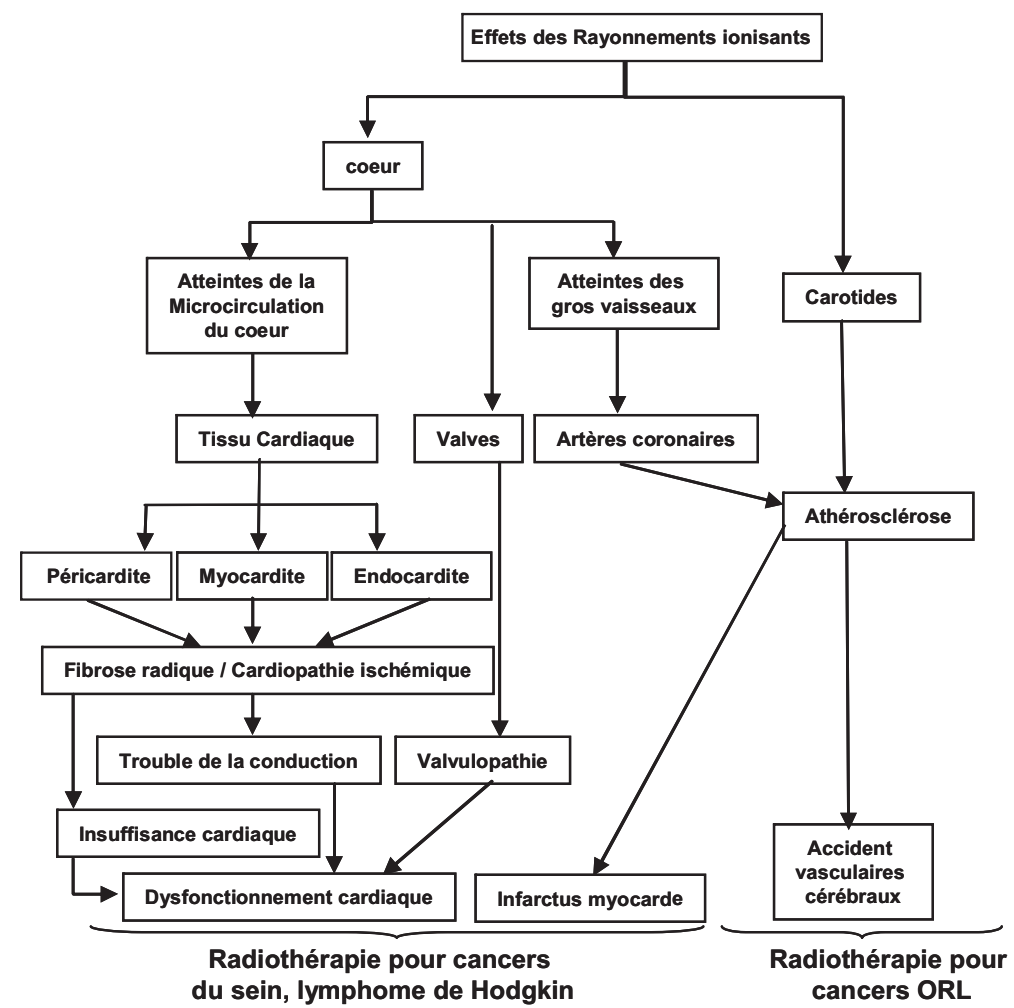

Figure 1-Représentation schématique des effets des rayonnements ionisants sur le système cardiovasculaire dans le cas des traitements par radiothérapie pour les cancers du sein, le lymphome de Hodgkin et les cancers de la sphère ORL.

Simplistic schema of ionising radiation effects on cardiovascular system after radiation therapy for breast cancer, Hodgkin's disease and head and neck cancer.

cancers de la sphère ORL et se manifestent par des accidents vasculaires cérébraux (AVC) (Gaugler et al., 2011).

En radiopathologie, le concept selon lequel une atteinte des microvaisseaux pourrait être à l'origine et l'élément crucial de la progression des lésions tissulaires radio-induites, contribuant à terme aux dysfonctionnements des organes dont le cœur, ne sera pas développé dans cette revue. Celle-ci portera sur les conséquences cardiovasculaires des irradiations thérapeutiques avec une attention particulière pour les atteintes des gros vaisseaux, consécutives à l'athérosclérose. Dans un second temps, les mécanismes moléculaires, biologiques et physiopathogiques 
étayés par des études expérimentales seront décrits. Enfin, les conséquences potentielles d'exposition à des faibles doses d'irradiation seront abordées.

\section{Radiothérapie et risque cardiovasculaire}

Environ 50 à $60 \%$ des patients atteints d'un cancer bénéficient d'une radiothérapie au cours de leur traitement. Bien que son efficacité thérapeutique soit indéniable, la radiothérapie entraine des dommages aux tissus sains présents dans le champ d'irradiation, avec comme conséquence d'éventuelles complications radioinduites à long terme (Bentzen et al., 2003).

À l'instar de l'athérosclérose liée à l'âge, l'athérosclérose radio-induite se développe insidieusement et de façon chronique. Le temps de latence entre l'irradiation et l'apparition des signes cliniques est de plusieurs années. Globalement, le risque relatif (RR) d'événements cardiovasculaires chez les patients irradiés est supérieur à celui de la population générale. Il est lié à des paramètres tels que : le nombre d'années suivant la radiothérapie, le champ d'irradiation (dose et volume), l'âge du patient au moment du traitement, la prédisposition génétique et les facteurs de co-morbidité vasculaire associés.

Des études montrent l'augmentation du risque cardiovasculaire avec la période de suivi après le traitement par radiothérapie. Sur plus de 100000 patientes traitées par radiothérapie pour un cancer du sein, le RR de mortalité cardiaque passe de 1,10 avant 10 ans à 1,53 après 15 ans pour les patientes ayant un cancer du sein gauche comparées aux patientes traitées pour un cancer du sein droit (Clarke et al., 2005 ; Darby et al., 2005 ; Demirci et al., 2009). Pour des patients jeunes traités pour un lymphome de Hodgkin par une radiothérapie supradiaphragmatique, le RR d'infarctus du myocarde par rapport à la population générale est multiplié par 2,4 après 10 ans de suivi, il atteint 4,3 au-delà de 20 ans (Swerdlow et al., 2007). Pour des patients de plus de 65 ans traités par radiothérapie pour un cancer de la tête et du cou, l'incidence des AVC passe de $19 \%$ à 5 ans à $34 \%$ à 10 ans (Smith et al., 2008). Avant 60 ans, le RR d'AVC passe de 3,7 pour un suivi postradiothérapie inférieur à 10 ans à 10,1 pour un suivi supérieur à 10 ans (Dorresteijn et al., 2002).

\section{Champ d'irradiation, dose, volume, âge, facteurs de risque traditionnels et risque cardiovasculaire}

L'influence du champ d'irradiation sur le risque cardiovasculaire est illustrée par le cas du cancer du sein. Une méta-analyse sur 40 essais cliniques randomisés (soit environ 20000 patientes) portant sur le bénéfice thérapeutique de la radiothérapie 
dans le traitement du cancer du sein montre une baisse de la mortalité chez les patientes traitées par radiothérapie (Early Breast Cancer Trialists' Collaborative Group, 2000). En revanche, cet effet favorable est contrebalancé par une augmentation du taux de mortalité annuel lié à des complications cardiovasculaires dues à l'irradiation des artères coronaires, des artères carotides ou d'autres artères principales. L'analyse de 63 essais randomisés incluant 32800 femmes traitées pour un cancer du sein montre que le taux de mortalité du aux pathologies cardiaques est $27 \%$ plus élevé chez les femmes traitées par radiothérapie que chez les femmes non traitées par radiothérapie (Clarke et al., 2005). La latéralité droite/gauche d'atteinte d'un cancer du sein n'influence pas l'utilisation ou non de la radiothérapie. Cependant, le risque de pathologies cardiovasculaires associées dépend du sein traité et les patientes ayant un cancer du sein gauche sont les plus exposées (Paszat et al., 1998). Vingt ans après la radiothérapie, les coronaropathies touchent $25 \%$ des patientes traitées pour un cancer du sein gauche contre $10 \%$ pour celles traitées pour un cancer du sein droit (Harris et al., 2006). Des études ont tenté d'estimer une dose seuil de tolérance et d'établir une relation dose/effet. Pour le cour, la dose de tolérance décrite est que le volume cardiaque recevant 35 Gy doit être inférieur à $30 \%$ et que la dose par fraction ne doit pas excéder $2 \mathrm{~Gy}$. L'établissement d'une relation dose/effet se heurte au problème de la pertinence d'estimation de la dose et de sa précision (Bolling and Willich, 2010), ainsi qu'à la détermination d'une incidence du rapport dose/volume sur le risque de complications cardiovasculaires tardives. La dose moyenne au cœur est estimée de 1 à 20 Gy en fonction de la latéralité de la tumeur et/ou de la technique utilisée (Taylor et al., 2007). L'évolution des techniques de radiothérapie implique que les irradiations de la sphère thoracique (cancer du sein ou du poumon) entrainent des doses au cœur hétérogènes et la prise en compte de la dose moyenne au cœur, du volume cardiaque exposé, de la région du cœur qui reçoit la dose maximum sont autant de paramètres qui influencent potentiellement le risque de pathologies cardiovasculaires. Une étude montre que pour des cancers du sein traités entre les années 1950 et 1990, la dose moyenne au cœur entier est de 0,9 à 14 Gy pour un cancer du sein gauche et de 0,4 à 6 Gy pour le traitement d'un cancer du sein droit (Taylor et al., 2007). L'irradiation des chaines mammaires internes engendrent des doses au cœur de 3 à 17 Gy pour un cancer du sein gauche et de 2 à $10 \mathrm{~Gy}$ pour un cancer du sein droit. La partie antérieure du cœur et l'artère inter-ventriculaire antérieure, site anatomique propice à l'athérosclérose pouvant provoquer des infarctus du myocarde, sont les zones anatomiques les plus exposées en terme de dose. Bien que la balistique se soit améliorée au cours de ces 50 dernières années et que les doses moyennes au cœur ainsi que celles reçues par l'artère interventriculaire antérieure aient considérablement diminuées, une étude montre que pour 50 patientes traitées en 2006 pour un cancer du sein gauche en GrandeBretagne, la dose moyenne au cœur entier est de 2,3 Gy, la dose reçue par l'artère 
inter-ventriculaire antérieure est de 7,6 Gy et, pour $44 \%$ des patientes, un volume de la partie antérieure du cœur reçoit plus de $20 \mathrm{~Gy}$ voire jusqu'à $30 \mathrm{~Gy}$ (Taylor et al., 2008). Une étude portant sur 4122 enfants atteints de cancers solides montre que le risque de mortalité cardiovasculaire à long terme (suivi moyen de 27 ans) est augmenté pour une dose moyenne estimée au cœur excédant $5 \mathrm{~Gy}$. Le RR est de 12,5 pour des doses moyennes estimées de 5 à 14,9 Gy et il est de 25,1 pour des doses supérieures à 15 Gy (Tukenova et al., 2010). Des études prospectives fondées sur des données dosimétriques précises (histogrammes dose/volume cœur entier et artères coronaires) avec un suivi à long terme des patients devraient permettre d'enrichir les connaissances dans ce domaine. De plus, les nouvelles techniques de radiothérapie (Tomothérapie, IMRT ou 3D-CRT) devraient réduire les doses délivrées au cœur mais dans des proportions variables en fonction des techniques (Coon et al., 2010).

Des complications cardiaques peuvent se développer indirectement suite à l'irradiation d'un autre organe à risque comme le poumon. Le traitement par radiothérapie du cancer du poumon et du lymphome de Hodgkin peut provoquer des pneumopathies aigues et des fibroses pulmonaires radio-induites (Tsoutsou and Koukourakis, 2006). Cinquante à $90 \%$ de ces patients présentent des tests anormaux de la fonctionnalité pulmonaire (McDonald et al., 1995) avec des insuffisances respiratoires plus ou moins sévères se manifestant par des dyspnées, des orthopnées et des cyanoses. De plus, le ventricule droit qui est en amont du poumon peut être atteint dans certains cas avec apparition d'une insuffisance cardiaque droite qui résulte de la dilatation ou de l'hypertrophie entre autres du ventricule droit (Seppenwoolde et al., 2004). Dans ce cas, l'établissement du risque cardiaque à long terme doit prendre en compte les doses de tolérance aux poumons associées aux histogrammes dose/volume de cet organe à risque.

Concernant les cancers de la tête et du cou, il n'existe pas de données sur la dose de tolérance des artères carotides ni de relation dose-réponse sur la survenue d'AVC. Les cancers du cou (carcinomes du larynx et de la parotide, adénome pléomorphe) sont traités par des doses totales importantes de 50 à 70 Gy délivrées par fraction de $2 \mathrm{~Gy}$. Ce traitement est associé à un risque accru d'AVC du aux atteintes carotidiennes (Dorresteijn et al., 2002).

L'âge du patient au moment du traitement influence le risque cardiovasculaire. Plus le patient est jeune, plus le risque est élevé. Dans une étude sur 39000 patients traités pour un cancer du testicule entre 1943 et 2001, le RR de mortalité cardiaque comparé à la population générale n'est significativement plus élevé que pour les patients âgés de moins de 35 ans au moment de la radiothérapie ( $R R=1,7)$ (Fossa et al., 2007). Une autre étude montre que chez 3462 patients traités par une radiothérapie supradiaphragmatique pour un lymphome de Hodgkin, le RR 
d'infarctus du myocarde comparé à la population générale est de 10,1 pour les moins de 35 ans au moment du traitement, de 2,9 pour les $35-54$ ans et de 1,8 pour les plus 55 ans (Swerdlow et al., 2007). Pour les cancers de la tête et du cou traités entre 1977 et 1998, les patients de plus de 50 ans au moment de la radiothérapie ont un RR d'AVC de 4,5 par rapport à la population générale et ce RR passe à 9,8 pour les moins de 50 ans. (Dorresteijn et al., 2002).

Le risque cardiovasculaire chez les patients irradiés est majoré en fonction d'autres facteurs de risque favorisant l'athérosclérose liée à l'âge comme l'hypercholestérolémie, l'hypertension, le diabète, et le tabagisme mais aussi par des antécédents de cardiopathies ischémiques ou la prise d'agents chimiothérapeutiques. Dans une étude sur 4414 patientes survivantes 10 ans après un cancer du sein traité entre 1970 à 1986, le RR d'infarctus du myocarde est de 1,36 pour le tabac, de 1,34 pour la radiothérapie et de 3,04 pour l'association des deux démontrant une interaction entre le tabac et la radiothérapie (Hooning et al., 2007). Dans cette même étude, l'hypercholestérolémie, l'hypertension et des antécédents de cardiopathies ischémiques majorent respectivement le RR d'un facteur $2,9,1,7$, et 1,5 . Pour les cancers de la tête et du cou, le RR d'AVC est majoré d'un facteur 2,1 et 2,9 respectivement pour les patients diabétiques ou souffrants d'hypertension traités par radiothérapie. Des patients ayant une chimiothérapie adjuvante à la radiothérapie (cyclophosphamide, methotrexate, fluorouracil) ont un risque accru comparé à ceux ayant reçus une radiothérapie seule (Hooning et al., 2007). Les anthracyclines souvent utilisées dans le traitement du cancer du sein ont une toxicité cardiaque intrinsèque et leur association avec la radiothérapie augmente le risque de pathologie cardiaque (Shapiro et al., 1998).

\section{Effets des rayonnements ionisants et pathologies cardiovasculaires : aspects physiopathologiques et mécanismes moléculaires}

Les données d'imagerie obtenues chez l'homme montre que l'athérosclérose radio-induite présente des spécificités comparée à l'athérosclérose liée à l'âge. Des clichés d'artériographie carotidienne réalisés chez des patients traités par radiothérapie pour un cancer de la sphère ORL montrent une apparition des lésions chez des patients plus jeunes avec une longueur des sténoses plus importante que dans une population contrôle (Silverberg et al., 1978 ; Houdart et al., 2001). Alors que les lésions carotidiennes classiques sont souvent associées à des atteintes diffuses et disséminées sur l'arbre vasculaire, les lésions carotidiennes radioinduites sont confinées au site irradié (Dorresteijn et al., 2005). Ces lésions sont localisées préférentiellement et de façon atypique dans l'artère carotide commune alors que les lésions classiques ont une localisation privilégiée dans l'artère 
carotide interne. Il n'y a pas d'atteinte du bulbe carotidien, contrairement aux lésions classiques (Modrall and Sadjadi, 2003). Une fibrose radio-induite se développe au niveau péri-artériel et des tissus sains environnant l'artère carotide irradiée (Houdart et al., 2001). Pour un degré de sténose identique, l'analyse par écho-doppler d'artères carotides irradiées montre une augmentation de l'épaisseur intima-média et une lumière intimale plus rétrécie comparativement à des artères carotides contrôles non irradiées (Cheng et al., 1999 ; Dorresteijn et al., 2005). L'examen angiographique d'artères coronaires irradiées montre que les sténoses coronariennes radio-induites sont plus souvent proximales et ostiales plutôt que distales (Veinot and Edwards, 1996).

$\mathrm{Au}$ niveau anatomopathologique, il est difficile de distinguer les lésions vasculaires radiques des lésions d'athérosclérose classiques et aucun élément histologique n'est spécifique des lésions radio-induites (Fajardo, 2005). Quelques nuances ont été décrites et comparativement aux lésions d'athérosclérose liées à l'âge, les lésions athéromateuses radio-induites sont associés à une fibrose artérielle et péri-artérielle plus sévère avec moins de macrophages spumeux et de cristaux de cholestérol. Des comparaisons des lésions radio-induites d'artères coronaires de patients traités pour un lymphome de Hodgkin et de patients non irradiés montrent que l'irradiation induit une fibrose plus étendue et plus sévère de l'intima et de l'adventice associé à un amincissement voire une destruction focalisée de la média (Veinot and Edwards, 1996 ; Miltenyi et al., 2004 ; SenkusKonefka and Jassem, 2007). Des augmentations des contenus en protéoglycanes dans l'intima et en cellules inflammatoires CD45+ dans la média ont également été décrites (Russell et al., 2009).

Les mécanismes moléculaires et cellulaires impliqués dans les lésions vasculaires induites par de fortes doses d'irradiation restent encore très mal connus (Stewart et al., 2006 ; Milliat et al., 2008a). Les difficultés méthodologiques pour mettre en place des modèles pertinents d'irradiation localisée et fractionnée à fortes doses expliquent le peu d'études précliniques s'intéressant au processus d'athérosclérose radio-induite. À l'exception d'une étude (Hoving et al., 2008), les quelques résultats précliniques sont obtenus sur des modèles d'irradiations à dose unique ne modélisant pas le fractionnement de dose réalisé chez l'homme en clinique. Cottin et al. ont montré que l'irradiation localisée d'artères de lapins hypercholestérolémiques induit une accélération de la formation de plaques d'athérosclérose (Cottin et al., 2001). Les rayonnements ionisants accélèrent aussi la formation des lésions aortiques chez des souris soumises à un régime proathérogène (Tribble et al., 1999). Ces lésions athérosclérotiques sont moins importantes dans des souris transgéniques surexprimant la superoxyde dismutase $(\mathrm{CuZn}-\mathrm{SOD})$, démontrant un rôle clef des espèces réactives de l'oxygène dans l'induction du processus pro-athérogène radio-induit (Tribble et al., 1999). Des 
études récentes chez des souris ApoE-/- prédisposées à l'athérosclérose montrent que l'irradiation accélère le développement des lésions d'athérosclérose après une dose unique de $14 \mathrm{~Gy}$ au niveau des artères carotides (Stewart et al., 2006 ; Hoving et al., 2008). Ces souris ApoE-/- irradiées présentent plus de plaques d'athérome et un noyau lipidique par plaque de plus grande taille comparé aux souris ApoE-/non irradiées d'âge équivalent. L'analyse des plaques d'athérome montre une forte implication des cellules inflammatoires notamment des macrophages et des granulocytes. La quantité de collagène dans ces plaques est diminuée après irradiation suggérant leur instabilité. Des macrophages ayant phagocyté des érythrocytes, présents exclusivement dans les plaques d'athérome d'artères carotides irradiées, reflètent un processus hémorragique. Ces plaques sont riches en dépôts de fibrine suggérant l'induction par l'irradiation de processus thrombotiques persistants. L'ensemble de ces observations démontre la spécificité phénotypique des lésions d'athérosclérose radio-induites et rend ainsi ce modèle très pertinent pour étudier la pathogenèse des évènements moléculaires et cellulaires radio-induits au niveau des artères et pour tester des approches pharmacologiques.

Les études in vitro ont permis de dégager un concept selon lequel l'irradiation induit une activation chronique de l'endothélium contribuant aux lésions vasculaires tardives. Cependant, les mécanismes décrits sont obtenus sur des cellules endothéliales de microvaisseaux ou sur des petits vaisseaux d'organes irradiés. L'extrapolation de ces résultats aux plus gros vaisseaux doit être faite avec prudence. Ces études montrent qu'une seule dose d'irradiation induit une activation ou dysfonction endothéliale aiguë perdurant plusieurs semaines après l'irradiation et entraînant un phénotype endothélial pro-inflammatoire, prothrombotique, procoagulant et antifibrinolytique (Gaugler et al., 1997 ; Gaugler, 2005 ; Milliat et al., 2008a). L'activation radio-induite de l'endothélium vasculaire se traduit par une augmentation maintenue dans le temps de l'expression des molécules d'adhésion participant au recrutement des cellules circulantes. Des surexpressions de VCAM1, d'ICAM-1, de PECAM-1, et des E- et P-sélectines ont été décrites in vitro sur des cellules endothéliales irradiées (Gaugler et al., 1997 ; Heckmann et al., 1998 ; Quarmby et al., 1999, 2000 ; Gaugler et al., 2004) favorisant les interactions plaquettes/endothélium et leucocytes/endothélium (Gaugler et al., 1997 ; VereyckenHoller et al., 2002 ; Mouthon et al., 2003 ; Gaugler et al., 2004, 2005). In vitro, cette augmentation des interactions plaquettes/endothélium est inhibée en présence d'un anticorps anti-PECAM-1 démontrant le rôle clef de cette molécule d'adhésion dans l'adhésion et l'agrégation plaquettaire sur des cellules endothéliales irradiées (Gaugler et al., 2004).

Les cellules endothéliales jouent aussi un rôle déterminant dans le contrôle du tonus vasculaire en sécrétant des vasoconstricteurs (endothéline, prostaglandines, 
thromboxane A2 angiotensine II) et des vasodilatateurs (prostacycline, monoxyde d'azote NO) agissant sur les cellules musculaires lisses (CML) vasculaires. Le $\mathrm{NO}^{\circ}$, principalement synthétisé par la NO-Synthase endothéliale (eNOS), a une action vasodilatatrice. Une baisse de l'expression de la eNOS a été décrite dans l'endothélium dans des modèles précliniques mais également chez l'homme au niveau de l'artère cervicale suite à une radiothérapie pour un cancer du cou (Sugihara et al., 1999).

L'activation du système de coagulation fait partie des processus physiologiques stimulés après une irradiation. La perte radio-induite de la thromborésistance endothéliale résulte de la stimulation de la fibrinogénèse et d'une baisse de la fibrinolyse associées à des surexpressions du facteur tissulaire (Verheij et al., 1995), du facteur von Willebrand (Jahroudi et al., 1996 ; Verheij et al., 1997), de l'inhibiteur des activateurs du plasminogène de type 1 (PAI-1) (Milliat et al., 2008b), et à des diminutions d'expression de la prostacycline (Rubin et al., 1985) et de la thrombomoduline (Zhou et al., 1992). La baisse de la thrombomoduline et la surexpression de PAI-1 au niveau de l'endothélium des vaisseaux de l'intestin ont été confirmées chez l'homme plusieurs semaines après radiothérapie (Richter et al., 1997 ; Milliat et al., 2008b).

En plus de leur capacité à s'activer en réponse à un stress radio-induit, les cellules endothéliales sont également capables d'influencer, par des effets paracrines, le phénotype des cellules présentes dans leur microenvironnement comme les cellules musculaires lisses vasculaires (Milliat et al., 2006). Les communications endothélium/CMLv sont essentielles dans l'initiation des dommages vasculaires dans les pathologies comme l'athérosclérose, l'hyperplasie néointimale ou l'hypertension (Peiro et al., 1995). Dans le contexte de l'irradiation, il a été montré que les cellules endothéliales irradiées stimulent la prolifération, la migration, et le phénotype pro-fibrosant des CML vasculaires (Milliat et al., 2006), suggérant le rôle des relations cellules endothéliales/CML vasculaires dans le développement des lésions vasculaires radio-induites.

Le concept d'activation radio-induite chronique de l'endothélium démontré expérimentalement sur des microvaisseaux a été confirmé très récemment en clinique sur des artères irradiées de patients traités pour un cancer de la tête et du cou (Halle et al., 2010, 2011). Chez ces patients, des biopsies d'artères irradiées ou non ont été prélevées de 4 semaines à 10 ans post-radiothérapie (dose moyenne prescrite de 50 à $64 \mathrm{~Gy}$ ). Une étude transcriptomique réalisée avec des puces à $\mathrm{ADN}$ montre des surexpressions de nombreux gènes pro-inflammatoires dont l'IL6, l'IL-8, l'IL-1 $\beta$, ICAM-1, VCAM-1, et la E-Sélectine dans les artères irradiées comparées aux artères non irradiées. Ces gènes sont régulés par la voie de signalisation pro-inflammatoire dépendant du facteur de transcription NF-KB. 
L'activation de NF- $\mathrm{BB}$ a été confirmée dans les artères irradiées par immunohistochimie avec la détection de la sous-unité p65 (Halle et al., 2010). Comme démontré in vitro sur des cellules endothéliales (Gaugler et al., 1997, 2004, 2005 ; Chou et al., 2009), l' activation radio-induite décrite dans cette étude (Halle et al., 2010) est persistante puisqu'elle est observée des mois voire des années post-radiothérapie. Ce travail souligne la pertinence de certains résultats obtenus des années auparavant sur des modèles expérimentaux simplifiés et illustre le potentiel d'une recherche fondamentale en amont débouchant sur des concepts pouvant être confirmés ensuite chez l'homme.

\section{Effets des rayonnements et pathologies cardiovasculaires : cas des doses moyennes et faibles}

Comme décrit dans les chapitres précédents, pour des niveaux de dose estimés de $5 \mathrm{~Gy}$ à $30 \mathrm{~Gy}$ voire plus il y a un risque de pathologies cardiovasculaires. Pour des niveaux d'exposition plus faibles ou très faibles, les données épidémiologiques sont moins nombreuses, contradictoires, parfois peu fiables en raison des biais associés aux cohortes (autres facteurs de risque cardiovasculaires), et/ou la taille des cohortes, et/ou des données dosimétriques associées très approximatives voire inexistantes (McGale and Darby, 2005 ; Metz-Flamant et al., 2009). Malgré tout, il existe une étude robuste portant sur 90000 manipulateurs en radiologie ayant débutés leur travail entre 1926 et 1982 montrant un risque de mortalité cardiovasculaire plus élevé pour les personnes ayant débutées avant 1940 par rapport à celles ayant débutées après 1960 (Hauptmann et al., 2003). Dans ce travail, en l'absence de données dosimétriques, l'estimation de la dose reçue a été réalisée par approximation en fonction de l'année du début de la pratique et des caractéristiques des postes occupés. Le peu d'études avec des données dosimétriques montrent un lien entre des irradiations à doses faibles (inférieures à 5 Gy) et un risque cardiovasculaire. La principale concerne le suivi épidémiologique (avec un recul de 55 ans) des survivants des bombardements atomiques d'Hiroshima et de Nagasaki au Japon (Life Span Study) (Yamada et al., 2004 ; Shimizu et al., 2010). Sur une population de plus de 86000 survivants ayant reçus une irradiation corps-entier uniforme pour une gamme de dose estimée entre 0 et 4 Gy (dose approximée par la dose reçue au colon), il y a un lien entre les risques de mortalité cardiovasculaire et d'AVC et la dose reçue. La relation doseréponse a été étudiée et le risque de mortalité cardiovasculaire augmente de $14 \%$ par Gy avec une incertitude pour des doses inférieures à $0.5 \mathrm{~Gy}$. Pour ces dernières, une étude réalisée en Grande Bretagne sur 64937 travailleurs du nucléaire entre 1946 et 2005 avec un suivi dosimétrique personnalisé (films dosimétriques) a permis de mettre en évidence un excès de risque de mortalité de pathologies 
cardiovasculaires (cardiopathies ischémiques) avec une relation dose/effet pour des doses cumulées comprises entre 0,1 et 0,4 Gy (McGeoghegan et al., 2008).

Comme mentionné dans le chapitre précédent, les mécanismes moléculaires et cellulaires impliqués dans les lésions vasculaires radio-induites aux fortes doses d'irradiation restent encore mal connus. Pour les faibles doses d'irradiation, il n'existe qu'un nombre anecdotique d'études expérimentales avec des résultats contradictoires. Quelques études in vitro montrent que, pour des doses inférieures à $5 \mathrm{~Gy}$, les cellules endothéliales surexpriment des molécules d'adhésion, le facteur von Willebrand, le PAI-1, le TGF- $\beta 1$ ou encore les cytokines proinflammatoires telles que 1'IL-6 ou l'IL-8 (Gaugler et al., 1997, 2005 ; Heckmann et al., 1998 ; Quarmby et al., 1999 ; Milliat et al., 2006, 2008b). Ces résultats vont dans le sens de ceux obtenus pour des doses d'irradiation plus fortes et suggèrent un effet pro-inflammatoire et pro-thrombotique dépendant de la dose d'irradiation. Cependant, certaines équipes décrivent un effet anti-inflammatoire pour des faibles voire des très faibles doses d'irradiation. Cet effet protecteur et antiinflammatoire est connu depuis longtemps et est utilisé en clinique pour traiter des pathologies bénignes des articulations douloureuses (arthrites, tendinites, bursites, inflammation de la membrane synoviale) (Seegenschmiedt et al., 2004). Il serait dû à une baisse des interactions des leucocytes avec l'endothélium et à une baisse de production des cytokines pro-inflammatoires (Rodel et al., 2007, 2008). In vivo, dans un modèle de cicatrisation cutanée chez des rats diabétiques, des doses de 0,075 Gy répétées pendant 5 à 15 jours, permettent d'accélérer la cicatrisation cutanée via la mobilisation des cellules de la moelle (Guo et al., 2010). Une exposition de souris à de très faibles doses $(0,025$ Gy tous les 2 jours pendant 2 à 16 semaines) permet d'atténuer l'inflammation cardiaque et la dysfonction rénale induite par la streptozotocine (Zhang et al., 2009, 2011). Enfin, dans un modèle d'ischémie de la patte chez la souris, l'exposition à une dose unique de 2 Gy permet de stimuler la régénération vasculaire en stimulant l'angiogénèse (Heissig et al., 2005).

Au-delà de ces quelques résultats, et à l'exception d'une étude très récente (Mitchel et al., 2011), il n'existe pas d'études expérimentales in vivo permettant de relier une exposition à des faibles doses d'irradiation avec la survenue ou l'accélération d'une pathologie vasculaire. Le travail de Mitchel et al., montre un effet soit protecteur soit délétère de faibles doses uniques corps entier d'irradiation (entre 0.025 et $0.5 \mathrm{~Gy}$ ) sur le développement de l'athérosclérose dans l'aorte de souris ApoE-/- prédisposées à l'athérosclérose. L'apparente dichotomie d'effet de l'irradiation pour une même dose totale d'irradiation réside dans le débit de dose. Un faible débit de dose est protecteur alors qu'un débit de dose plus important est délétère. Cette étude démontre aussi que la relation dose/effet obtenue aux fortes doses ne peut pas être extrapolée aux faibles doses et que, dans ce modèle 
expérimental et dans cette gamme de dose, il n'y a pas de lien entre la dose et l'effet. La méthodologie expérimentale mise en place pour répondre aux questions des fortes doses ne peut pas simplement se transposer à la méthodologie qu'il faudra développer spécifiquement pour répondre à la problématique d'expositions chroniques à de faibles doses et à des débits de dose variables.

\section{Conclusion}

Concernant les fortes doses d'exposition, l'évolution des techniques de radiothérapie avec l'amélioration de la balistique devrait permettre de réduire les volumes et les doses aux organes à risques présents dans le champ d'irradiation. Cependant, en dépit de ces avancées, le cœur, les artères coronaires et les artères carotides ne peuvent pas être complètement exclus et les niveaux de doses reçus par ces organes restent et resteront importants (Coon et al., 2010). Des études épidémiologiques prospectives s'appuyant sur des données dosimétriques précises permettront à moyen terme de déterminer le risque personnalisé de chaque patient et de proposer un suivi cardiologique adapté voire une prise en charge thérapeutique pour prévenir l'évolution des pathologies cardiovasculaires. Concernant les programmes de recherche, des efforts substantiels devront être mis en œuvre afin de faire avancer les connaissances sur la pathologie cardiovasculaire radio-induite au sens large. La mise en place de modèles précliniques pertinents, adaptés aux questions posées, avec des configurations d'irradiation très ciblées (van Luijk et al., 2007) et l'utilisation de modèles transgéniques innovants sont des paramètres à considérer.

Pour les faibles doses d'exposition, il faudra également prendre en compte dans le domaine médical un suivi épidémiologique des personnes exposées suite à des examens d'imagerie médicale à visée diagnostique ou suite à des actes chirurgicaux assistés par imagerie. Aux États-Unis, on estime qu'entre 1995 et 2005, l'utilisation des techniques d'imagerie utilisant les rayonnements ionisants a été multipliée par quatre et qu'entre 1980 et 2006, la dose cumulée due aux expositions à visée diagnostique a été multipliée par 7 (Mettler et al., 2008 ; Gerber et al., 2009). Enfin, une des questions importantes en radiopathologie ces prochaines années sera de savoir si les potentiels effets cardiovasculaires aux faibles doses d'irradiation sont associés à une relation dose-effet, et dans ce cas, il faudra définir au mieux la dose-seuil induisant ces effets déterministes. À l'instar des effets des rayonnements ionisants sur le développement des cancers, on ne peut exclure le fait que les effets cardiovasculaires après exposition à des faibles doses d'irradiation soient liés à des phénomènes stochastiques sans effet de seuil. Des avancées scientifiques sur ces sujets influenceront les futures normes de radioprotection et notre vision de la radioprotection médicale elle-même. 


\section{RÉFÉRENCES}

Bentzen S.M., Dorr W., Anscher M.S., Denham J.W., Hauer-Jensen M., Marks L.B., Williams J. (2003) Normal tissue effects: Reporting and analysis, Semin. Radiat. Oncol. 13, 189-202.

Bolling T., Willich N. (2010) Long-term overall and cardiovascular mortality after childhood cancer: The problem of retrospective estimated radiation doses, J. Clin. Oncol. 28, e436; author reply e437-438.

Cheng S.W., Wu L.L., Ting A.C., Lau H., Lam L.K., Wei W.I. (1999) Irradiation-induced extracranial carotid stenosis in patients with head and neck malignancies, Am. J. Surg. 178, 323-328.

Chou C.H., Chen S.U., Cheng J.C. (2009) Radiation-induced interleukin-6 expression through mapk/p38/nf-kappab signaling pathway and the resultant antiapoptotic effect on endothelial cells through mcl-1 expression with sil6-ralpha, Int. J. Radiat. Oncol. Biol. Phys. 75, 1553-1561.

Clarke M., Collins R., Darby S., Davies C., Elphinstone P., Evans E., Godwin J., Gray R., Hicks C., James S. and others (2005) Effects of radiotherapy and of differences in the extent of surgery for early breast cancer on local recurrence and 15-year survival: An overview of the randomised trials, Lancet 366, 2087-2106.

Coon A.B., Dickler A., Kirk M.C., Liao Y., Shah A.P., Strauss J.B., Chen S., Turian J., Griem K.L. (2010) Tomotherapy and multifield intensity-modulated radiotherapy planning reduce cardiac doses in left-sided breast cancer patients with unfavorable cardiac anatomy, Int. J. Radiat. Oncol. Biol. Phys. 78, 104-110.

Cottin Y., Kollum M., Kolodgie F.D., Chan R.C., Kim H.S., Vodovotz Y., Virmani R., Waksman R., Yazdi H. (2001) Intravascular radiation accelerates atherosclerotic lesion formation of hypercholesteremic rabbits, Cardiovasc. Radiat. Med. 2, 231-240.

Darby S.C., McGale P., Taylor C.W., Peto R. (2005) Long-term mortality from heart disease and lung cancer after radiotherapy for early breast cancer: Prospective cohort study of about 300,000 women in us seer cancer registries, Lancet Oncol. 6, 557-565.

Demirci S., Nam J., Hubbs J.L., Nguyen T., Marks L.B. (2009) Radiation-induced cardiac toxicity after therapy for breast cancer: Interaction between treatment era and follow-up duration, Int. J. Radiat. Oncol. Biol. Phys. 73, 980-987.

Dorresteijn L.D., Kappelle A.C., Boogerd W., Klokman W.J., Balm A.J., Keus R.B., van Leeuwen F.E., Bartelink H. (2002) Increased risk of ischemic stroke after radiotherapy on the neck in patients younger than 60 years, J. Clin. Oncol. 20, 282-288.

Dorresteijn L.D., Kappelle A.C., Scholz N.M., Munneke M., Scholma J.T., Balm A.J., Bartelink H., Boogerd W. (2005) Increased carotid wall thickening after radiotherapy on the neck, Eur. J. Cancer 41, 1026-1030.

Early Breast Cancer Trialists' Collaborative Group (EBCTCG) (2000) Favourable and unfavourable effects on long-term survival of radiotherapy for early breast cancer: An overview of the randomised trials. Early breast cancer trialists' collaborative group, Lancet 355, 1757-1770.

Fajardo L.F. (2005) The pathology of ionizing radiation as defined by morphologic patterns, Acta Oncol. 44, 13-22.

Fossa S.D., Gilbert E., Dores G.M., Chen J., McGlynn K.A., Schonfeld S., Storm H., Hall P., Holowaty E., Andersen A. and others (2007) Noncancer causes of death in survivors of testicular cancer, J. Natl. Cancer. Inst. 99, 533-544.

Gaugler M.H. (2005) A unifying system: Does the vascular endothelium have a role to play in multiorgan failure following radiation exposure? BJR Suppl. 27, 100-105.

Gaugler M.H., Drouet F., Krempf M. (2011) radiotherapy and atherosclerosis: Current data and issues, Med. Sci. (Paris) 26, 740-746.

Gaugler M.H., Squiban C., van der Meeren A., Bertho J.M., Vandamme M., Mouthon M.A. (1997) Late and persistent up-regulation of intercellular adhesion molecule-1 (icam-1) expression by ionizing radiation in human endothelial cells in vitro, Int. J. Radiat. Biol. 72, 201-209. 


\section{LES EFFETS DES RAYONNEMENTS IONISANTS SUR LE SYSTÈME CARDIOVASCULAIRE}

Gaugler M.H., Vereycken-Holler V., Squiban C., Aigueperse J. (2004) Pecam-1 (cd31) is required for interactions of platelets with endothelial cells after irradiation, J. Thromb. Haemost. 2, 20202026

Gaugler M.H., Vereycken-Holler V., Squiban C., Vandamme M., Vozenin-Brotons M.C., Benderitter M. (2005) Pravastatin limits endothelial activation after irradiation and decreases the resulting inflammatory and thrombotic responses, Radiat. Res. 163, 479-487.

Gerber T.C., Carr J.J., Arai A.E., Dixon R.L., Ferrari V.A., Gomes A.S., Heller G.V., McCollough C.H., McNitt-Gray M.F., Mettler F.A. et al. (2009) Ionizing radiation in cardiac imaging: A science advisory from the american heart association committee on cardiac imaging of the council on clinical cardiology and committee on cardiovascular imaging and intervention of the council on cardiovascular radiology and intervention, Circulation 119, 1056-1065.

Guo W.Y., Wang G.J., Wang P., Chen Q., Tan Y., Cai L. (2010) Acceleration of diabetic wound healing by low-dose radiation is associated with peripheral mobilization of bone marrow stem cells, Radiat. Res. 174, 467-479.

Halle M., Gabrielsen A., Paulsson-Berne G., Gahm C., Agardh H.E., Farnebo F., Tornvall P. (2010) Sustained inflammation due to nuclear factor-kappa b activation in irradiated human arteries, $J$. Am. Coll. Cardiol. 55, 1227-1236.

Halle M., Hall P., Tornvall P. (2011) Cardiovascular disease associated with radiotherapy: Activation of nuclear factor kappa-b, J. Int. Med. 269, 469-477.

Harris E.E., Correa C., Hwang W.T., Liao J., Litt H.I., Ferrari V.A., Solin L.J. (2006) Late cardiac mortality and morbidity in early-stage breast cancer patients after breast-conservation treatment, J. Clin. Oncol. 24, 4100-4106.

Hauptmann M., Mohan A.K., Doody M.M., Linet M.S., Mabuchi K. (2003) Mortality from diseases of the circulatory system in radiologic technologists in the united states, Am. J. Epidemiol. 157, 239-248.

Heckmann M., Douwes K., Peter R., Degitz K. (1998) Vascular activation of adhesion molecule mrna and cell surface expression by ionizing radiation, Exp. Cell. Res. 238, 148-154.

Heidenreich P.A., Kapoor J.R. (2009) Radiation induced heart disease: Systemic disorders in heart disease, Heart 95, 252-258.

Heissig B., Rafii S., Akiyama H., Ohki Y., Sato Y., Rafael T., Zhu Z., Hicklin D.J., Okumura K., Ogawa H. et al. (2005) Low-dose irradiation promotes tissue revascularization through vegf release from mast cells and mmp-9-mediated progenitor cell mobilization, J. Exp. Med. 202, 739-750.

Hooning M.J., Botma A., Aleman B.M., Baaijens M.H., Bartelink H., Klijn J.G., Taylor C.W., van Leeuwen F.E. (2007) Long-term risk of cardiovascular disease in 10-year survivors of breast cancer, J. Natl. Cancer Inst. 99, 365-375.

Houdart E., Mounayer C., Chapot R., Saint-Maurice J.P., Merland J.J. (2001) Carotid stenting for radiation-induced stenoses: A report of 7 cases, Stroke 32, 118-121.

Hoving S., Heeneman S., Gijbels M.J., te Poele J.A., Russell N.S., Daemen M.J., Stewart F.A. (2008) Single-dose and fractionated irradiation promote initiation and progression of atherosclerosis and induce an inflammatory plaque phenotype in apoe(-/-) mice, Int. J. Radiat. Oncol. Biol. Phys. 71, 848-857.

Jahroudi N., Ardekani A.M., Greenberger J.S. (1996) Ionizing irradiation increases transcription of the von willebrand factor gene in endothelial cells, Blood 88, 3801-3814.

Little M.P., Tawn E.J., Tzoulaki I., Wakeford R., Hildebrandt G., Paris F., Tapio S., Elliott P. (2008) A systematic review of epidemiological associations between low and moderate doses of ionizing radiation and late cardiovascular effects, and their possible mechanisms, Radiat. Res. 169, 99-109. 
Little M.P., Tawn E.J., Tzoulaki I., Wakeford R., Hildebrandt G., Paris F., Tapio S., Elliott P. (2010) Review and meta-analysis of epidemiological associations between low/moderate doses of ionizing radiation and circulatory disease risks, and their possible mechanisms, Radiat. Environ. Biophys. 49, 139-153.

McDonald S., Rubin P., Phillips T.L., Marks L.B. (1995) Injury to the lung from cancer therapy: Clinical syndromes, measurable endpoints, and potential scoring systems, Int. J. Radiat. Oncol. Biol. Phys. 31, 1187-1203.

McGale P., Darby S.C. (2005) Low doses of ionizing radiation and circulatory diseases: A systematic review of the published epidemiological evidence, Radiat. Res. 163, 247-257.

McGeoghegan D., Binks K., Gillies M., Jones S., Whaley S. (2008) The non-cancer mortality experience of male workers at british nuclear fuels plc, 1946-2005, Int. J. Epidemiol. 37, 506-518.

Mettler F.A. Jr., Thomadsen B.R., Bhargavan M., Gilley D.B., Gray J.E., Lipoti J.A., McCrohan J., Yoshizumi T.T., Mahesh M. (2008) Medical radiation exposure in the u.S. In 2006: Preliminary results, Health. Phys. 95, 502-507.

Metz-Flamant C., Bonaventure A., Milliat F., Tirmarche M., Laurier D., Bernier M.O. (2009) [low doses of ionizing radiation and risk of cardiovascular disease: A review of epidemiological studies], Rev. Epidémiol. Santé Publique 57, 347-359.

Milliat F., Francois A., Isoir M. et al. (2006) Influence of endothelial cells on vascular smooth muscle cells phenotype after irradiation: Implication in radiation-induced vascular damages, Am. J. Pathol. 169, 1484-1495.

Milliat F., Francois A., Tamarat R., Benderitter M. (2008a) role of endothelium in radiation-induced normal tissue damages, Ann. Cardiol. Angeiol. (Paris) 57, 139-148.

Milliat F., Sabourin J.C., Tarlet G., Holler V., Deutsch E., Buard V., Tamarat R., Atfi A., Benderitter M., Francois A. (2008b) Essential role of plasminogen activator inhibitor type-1 in radiation enteropathy, Am. J. Pathol. 172, 691-701.

Miltenyi Z., Keresztes K., Garai I., Edes I., Galajda Z., Toth L., Illes A. (2004) Radiation-induced coronary artery disease in hodgkin's disease, Cardiovasc. Radiat. Med. 5, 38-43.

Mitchel R.E., Hasu M., Bugden M., Wyatt H., Little M.P., Gola A., Hildebrandt G., Priest N.D., Whitman S.C. (2011) Low-dose radiation exposure and atherosclerosis in apoe(-/-) mice, Radiat. Res. 175, 665-675.

Modrall J.G., Sadjadi J. (2003) Early and late presentations of radiation arteritis, Semin. Vasc. Surg. 16, 209-214.

Mouthon M.A., Vereycken-Holler V., Van der Meeren A., Gaugler M.H. (2003) Irradiation increases the interactions of platelets with the endothelium in vivo: Analysis by intravital microscopy, Radiat. Res. 160, 593-599.

Paszat L.F., Mackillop W.J., Groome P.A., Boyd C., Schulze K., Holowaty E. (1998) Mortality from myocardial infarction after adjuvant radiotherapy for breast cancer in the surveillance, epidemiology, and end-results cancer registries, J. Clin. Oncol. 16, 2625-2631.

Peiro C., Redondo J., Rodriguez-Martinez M.A., Angulo J., Marin J., Sanchez-Ferrer C.F. (1995) Influence of endothelium on cultured vascular smooth muscle cell proliferation, Hypertension 25, 748-751.

Quarmby S., Hunter R.D., Kumar S. (2000) Irradiation induced expression of cd31, icam-1 and vcam1 in human microvascular endothelial cells, Anticancer Res. 20, 3375-3381.

Quarmby S., Kumar P., Wang J., Macro J.A., Hutchinson J.J., Hunter R.D., Kumar S. (1999) Irradiation induces upregulation of cd31 in human endothelial cells, Arterioscler Thromb. Vasc. Biol. 19, 588-597.

Richter K.K., Fink L.M., Hughes B.M., Sung C.C., Hauer-Jensen M. (1997) Is the loss of endothelial thrombomodulin involved in the mechanism of chronicity in late radiation enteropathy? Radiother. Oncol. 44, 65-71. 


\section{LES EFFETS DES RAYONNEMENTS IONISANTS SUR LE SYSTÈME CARDIOVASCULAIRE}

Rodel F., Keilholz L., Herrmann M., Sauer R., Hildebrandt G. (2007) Radiobiological mechanisms in inflammatory diseases of low-dose radiation therapy, Int. J. Radiat. Biol. 83, 357-366.

Rodel F., Hofmann D., Auer J., Keilholz L., Rollinghoff M., Sauer R., Beuscher H.U. (2008) The antiinflammatory effect of low-dose radiation therapy involves a diminished ccl20 chemokine expression and granulocyte/endothelial cell adhesion, Strahlenther Onkol. 184, 41-47.

Ross R. (1999) Atherosclerosis - an inflammatory disease, N. Engl. J. Med. 340, 115-126.

Rubin D.B., Drab E.A., Ts'ao C.H., Gardner D., Ward W.F. (1985) Prostacyclin synthesis in irradiated endothelial cells cultured from bovine aorta, J. Appl. Physiol. 58, 592-597.

Russell N.S., Hoving S., Heeneman S., Hage J.J., Woerdeman L.A., de Bree R., Lohuis P.J., Smeele L., Cleutjens J., Valenkamp A. and others (2009) Novel insights into pathological changes in muscular arteries of radiotherapy patients, Radiother. Oncol. 92, 477-483.

Seegenschmiedt M.H., Micke O., Willich N. (2004) Radiation therapy for nonmalignant diseases in germany. Current concepts and future perspectives, Strahlenther Onkol. 180, 718-730.

Senkus-Konefka E., Jassem J. (2007) Cardiovascular effects of breast cancer radiotherapy, Cancer Treat. Rev. 33, 578-593.

Seppenwoolde Y., De Jaeger K., Boersma L.J., Belderbos J.S., Lebesque J.V. (2004) Regional differences in lung radiosensitivity after radiotherapy for non-small-cell lung cancer, Int. J. Radiat. Oncol. Biol. Phys. 60, 748-758.

Shapiro C.L., Hardenbergh P.H., Gelman R., Blanks D., Hauptman P., Recht A., Hayes D.F., Harris J., Henderson I.C. (1998) Cardiac effects of adjuvant doxorubicin and radiation therapy in breast cancer patients, J. Clin. Oncol. 16, 3493-3501.

Shimizu Y., Kodama K., Nishi N., Kasagi F., Suyama A., Soda M., Grant E.J., Sugiyama H., Sakata R., Moriwaki H. et al. (2010) Radiation exposure and circulatory disease risk: Hiroshima and Nagasaki atomic bomb survivor data, 1950-2003, BMJ 340, b5349.

Silverberg G.D., Britt R.H., Goffinet D.R. (1978) Radiation-induced carotid artery disease, Cancer 41, 130-137.

Smith G.L., Smith B.D., Buchholz T.A., Giordano S.H., Garden A.S., Woodward W.A., Krumholz H.M., Weber R.S., Ang K.K., Rosenthal D.I. (2008) Cerebrovascular disease risk in older head and neck cancer patients after radiotherapy, J. Clin. Oncol. 26, 5119-5125.

Stewart F.A., Heeneman S., Te Poele J., Kruse J., Russell N.S., Gijbels M., Daemen M. (2006) Ionizing radiation accelerates the development of atherosclerotic lesions in apoe-/- mice and predisposes to an inflammatory plaque phenotype prone to hemorrhage, Am. J. Pathol. 168, 649-658.

Sugihara T., Hattori Y., Yamamoto Y., Qi F., Ichikawa R., Sato A., Liu M.Y., Abe K., Kanno M. (1999) Preferential impairment of nitric oxide-mediated endothelium-dependent relaxation in human cervical arteries after irradiation, Circulation 100, 635-641.

Swerdlow A.J., Higgins C.D., Smith P., Cunningham D., Hancock B.W., Horwich A., Hoskin P.J., Lister A., Radford J.A., Rohatiner A.Z. and others (2007) Myocardial infarction mortality risk after treatment for hodgkin disease: A collaborative british cohort study, J. Natl. Cancer Inst. 99, 206-214.

Taylor C.W., Nisbet A., McGale P., Darby S.C. (2007) Cardiac exposures in breast cancer radiotherapy: 1950s-1990s, Int. J. Radiat. Oncol. Biol. Phys. 69, 1484-1495.

Taylor C.W., Povall J.M., McGale P., Nisbet A., Dodwell D., Smith J.T., Darby S.C. (2008) Cardiac dose from tangential breast cancer radiotherapy in the year 2006, Int. J. Radiat. Oncol. Biol. Phys. 72, 501-507.

Tribble D.L., Barcellos-Hoff M.H., Chu B.M., Gong E.L. (1999) Ionizing radiation accelerates aortic lesion formation in fat-fed mice via sod-inhibitable processes, Arterioscler Thromb. Vasc. Biol. 19, 1387-1392.

Tsoutsou P.G., Koukourakis M.I. (2006) Radiation pneumonitis and fibrosis: Mechanisms underlying its pathogenesis and implications for future research, Int. J. Radiat. Oncol. Biol. Phys. 66, 12811293. 
Tukenova M., Guibout C., Oberlin O., Doyon F., Mousannif A., Haddy N., Guerin S., Pacquement H., Aouba A., Hawkins M. and others (2010) Role of cancer treatment in long-term overall and cardiovascular mortality after childhood cancer, J. Clin. Oncol. 28, 1308-1315.

van Luijk P., Faber H., Meertens H., Schippers J.M., Langendijk J.A., Brandenburg S., Kampinga H.H., Coppes R.P. (2007) The impact of heart irradiation on dose-volume effects in the rat lung, Int. J. Radiat. Oncol. Biol. Phys. 69, 552-559.

Veinot J.P., Edwards W.D. (1996) Pathology of radiation-induced heart disease: A surgical and autopsy study of 27 cases, Hum. Pathol. 27, 766-773.

Vereycken-Holler V., Aigueperse J., Gaugler M.H. (2002) Radiation effects on circulating and endothelial cell interactions studied by quantitative real-time videomicroscopy, Int. J. Radiat. Biol. 78, 923-930.

Verheij M., Dewit L.G., van Mourik J.A. (1995) The effect of ionizing radiation on endothelial tissue factor activity and its cellular localization, Thromb. Haemost. 73, 894-895.

Verheij M., Dewit L., van Mourik J.A. (1997) Radiation-induced von willebrand factor release, Blood 90, 2109-2110.

Yamada M., Wong F.L., Fujiwara S., Akahoshi M., Suzuki G. (2004) Noncancer disease incidence in atomic bomb survivors, 1958-1998, Radiat. Res. 161, 622-632.

Zhang C., Tan Y., Guo W., Li C., Ji S., Li X., Cai L. (2009) Attenuation of diabetes-induced renal dysfunction by multiple exposures to low-dose radiation is associated with the suppression of systemic and renal inflammation, Am. J. Physiol. Endocrinol. Metab. 297, E1366-1377.

Zhang C., Jin S., Guo W., Li C., Li X., Rane M.J., Wang G., Cai L. (2011) Attenuation of diabetesinduced cardiac inflammation and pathological remodeling by low-dose radiation, Radiat. Res. 175, 307-321.

Zhou Q., Zhao Y., Li P., Bai X., Ruan C. (1992) Thrombomodulin as a marker of radiation-induced endothelial cell injury, Radiat. Res. 131, 285-289. 